\title{
Painful red eyes in a contact lens wearer
}

In a Case Review article (BMJ 2017;358:j3614, doi:10.1136/ bmj.j3614) by Katherine McVeigh and colleagues, the name of co-author Kaveh Vahdani was spelt incorrectly, and should be spelt as above. 